Discrete Comput Geom 32:435-436 (2004)

DOI: $10.1007 / \mathrm{s} 00454-004-1130-6$

\title{
Guest Editors' Preface
}

This special issue is dedicated to Louis J. Billera on the occasion of his 60th birthday.

Louis Billera received his Ph.D. from City University of New York in 1968 with a dissertation in game theory, formally under the direction of Moses Richardson, but with considerable help from Michel Balinski of CUNY and Michael Maschler of the Hebrew University. That year he began his long career at Cornell, where he is now a Professor of Mathematics, and a member of the Center for Applied Mathematics and the Graduate Field of Operations Research. His fellowships and leaves have taken him to Hebrew University, Brandeis University, Université Catholique de Louvain (Belgium), Rutgers University, Mittag-Leffler Institute (Sweden) and the Mathematical Sciences Research Institute (Berkeley). In 1989 he was the first Associate Director of the National Science Foundation Center for Discrete Mathematics and Theoretical Computer Science (DIMACS) at Rutgers University.

In his distinguished career Louis Billera has published in the areas of game theory, geometric combinatorics and algebraic combinatorics. In 1994 Louis Billera was awarded the Fulkerson Prize in Discrete Mathematics by the American Mathematical Society and the Mathematical Programming Society for his research in multivariate splines. His ground-breaking contributions include (but are not limited to):

- research on the allocation of shared costs (with David Heath)

- the use of decomposability of polyhedra in bounding diameter (with J. Scott Provan)

- the proof of the sufficiency of McMullen's conditions on $f$-vectors of simplicial polytopes (with Carl Lee),

- the generalized Dehn-Sommerville equations on flag vectors of polytopes (with Margaret Bayer),

- the combinatorial study of multivariate splines (with Ruth Haas and Lauren Rose),

- the introduction of fiber polytopes and further work on secondary polytopes (with Paul Filliman and Bernd Sturmfels),

- the proof of the monotonicity of the $c d$-index (with Richard Ehrenborg),

- the determination of the cone of flag vectors for graded posets (with Gabor Hetyei),

- the relationship of the algebra of flag-enumeration functionals on Eulerian posets to the algebra of peak quasisymmetric functions (with Samuel Hsiao and Stephanie van Willigenburg), and

- the introduction of a continuous model for the space of phylogenetic trees (with Susan Holmes and Karen Vogtmann). 
Contributors to this issue include two of Louis Billera's previous Ph.D. students (Kathryn Nyman and Lauren Rose), two other Cornell graduates who studied with him (Shmuel Onn and Jesús De Loera), two people who have had postdoctoral positions with Louis Billera at Cornell (Richard Ehrenborg and Stephanie van Willigenburg), one who is a colleague at Cornell (Edward Swartz), and four who have coauthored papers with him (Richard Ehrenborg, Gábor Hetyei, Lauren Rose and Stephanie van Willigenburg). The papers in this special issue reflect the breadth of Louis Billera's mathematical interests. Topics include Ferrers diagrams, geometric lattices, Eulerian posets, constructions of polytopes and convex bodies with special properties, mixed fiber polytopes, stresses on frameworks, splines, vector partition functions and convex combinatorial optimization.

Louis Billera has made a special contribution to the field through his wonderful mentoring of students, postdocs and other colleagues. Nineteen students (nine of them women!) have completed their Ph.D.s at Cornell under his supervision. We speak for many when we express our gratitude to Lou for the encouragement and support he has given us throughout our careers.

Margaret M. Bayer

University of Kansas

Carl W. Lee

University of Kentucky

Bernd Sturmfels

University of California Berkeley 